\title{
Associations Between General Perceptions of COVID-19 and Posttraumatic Stress Disorder in Korean Hospital Workers: Effect Modification by Previous Middle East Respiratory Syndrome Coronavirus Experience and Occupational Type
}

\author{
Youngrong Lee', Kwanghyun Kim' ', Sungjin Park², Sun Jae Jung ${ }^{1,3}$ \\ ${ }^{1}$ Department of Preventive Medicine, Yonsei University College of Medicine, Seoul, Korea; ${ }^{2}$ Department of Occupational and Environmental \\ Medicine, Cheonan Medical Center, Cheonan, Korea; ${ }^{3}$ Department of Epidemiology, Harvard T.H. Chan School of Public Health, Boston, MA, USA
}

Objectives: This study investigated associations between perceptions of coronavirus disease 2019 (COVID-19) and the prevalence of posttraumatic stress disorder (PTSD) in workers at hospitals designated to treat COVID-19, as well as the difference in the magnitude of these associations by occupational type and previous Middle East respiratory syndrome coronavirus (MERS-CoV) experience.

Methods: The participants were workers at hospitals designated to treat COVID-19 who completed a questionnaire about their perceptions related to COVID-19, work experience during the previous MERS-CoV outbreak, and symptoms of PTSD ascertained by the PTSD Checklist for the Diagnostic and Statistical Manual of Mental Disorders. Participants' characteristics were compared using the chi-square test. Multivariable logistic regression was performed to evaluate the associations between perceptions and the prevalence of PTSD, stratified by occupational type and previous MERS-CoV experience.

Results: Non-medical personnel showed stronger associations with PTSD than medical personnel according to general fear (odds ratio [OR], 6.67; 95\% confidence interval [Cl], 1.92 to 23.20$)$, shortages of supplies $(\mathrm{OR}, 1.29 ; 95 \% \mathrm{Cl}, 1.07$ to 1.56$)$, and issue-specific fear $(\mathrm{OR}, 1.29 ; 95 \% \mathrm{Cl}, 1.05$ to 1.59$)$. Those with prior MERS-CoV quarantine experience were more prone to PTSD than those without such experience in terms of general fear (OR, 1.70; $95 \% \mathrm{Cl}, 1.22$ to 2.37$)$, shortages of supplies $(\mathrm{OR}, 1.24 ; 95 \% \mathrm{Cl}, 1.10$ to 1.40$)$, and issuespecific fear $(\mathrm{OR}, 1.21 ; 95 \% \mathrm{Cl}, 1.06$ to 1.38$)$.

Conclusions: During the COVID-19 pandemic, non-medical personnel tended to have higher odds of being categorized as having PTSD. Workers with prior MERS-CoV experience were more susceptible than those without such experience. These findings suggest the need for timely interventions to manage human resources for a sustainable quarantine system.

Key words: SARS-CoV-2, Posttraumatic stress disorder, Health personnel, Middle East respiratory syndrome coronavirus, Hospital administration

Received: November 2, 2020 Accepted: January 5, 2021

Corresponding author: Sun Jae Jung

Department of Preventive Medicine, Yonsei University College of

Medicine, 50-1 Yonsei-ro, Seodaemun-gu, Seoul 03722, Korea

E-mail: sunjaejung@yuhs.ac

This is an Open Access article distributed under the terms of the Creative Commons Attribution Non-Commercial License (https://creativecommons.org/licenses/bync/4.0/) which permits unrestricted non-commercial use, distribution, and reproduction in any medium, provided the original work is properly cited.

\section{INTRODUCTION}

Ever since the first confirmed case [1] of severe acute respiratory syndrome coronavirus 2 (SARS-CoV-2) was reported, coronavirus disease 2019 (COVID-19) has triggered a significant amount of stress around the world [2]. People whose safety is threatened by sudden natural disasters or a massive outbreak of an emerging infectious disease like COVID-19 are at an elevated risk of suffering from posttraumatic stress disor- 
der (PTSD). Stress is likely to be severe for hospital workers, such as physicians, nurses, and other administrative staff, who are involved in the response to the pandemic [3-5].

The COVID-19 pandemic could expose healthcare professionals worldwide to unprecedented burdens entailing dilemmatic decisions, such as how to allocate scarce resources to equally needy patients; how to balance their own healthcare needs with those of patients; how to balance their responsibility toward the patients with that toward their family and relatives; and how to provide care for all severely ill patients with limited resources [6]. Healthcare staff, who are under extreme pressure, are at an increased risk of moral injury [7] and mental health problems as they deal with the challenges posed by the pandemic. As a previous example, during the 2003 outbreak of severe acute respiratory syndrome coronavirus (SARS(oV) in Taiwan, most of the staff at the emergency departments and psychiatric wards developed PTSD [8]. Furthermore, medical staff who performed tasks related to Middle East respiratory syndrome (MERS) showed PTSD symptoms after the outbreak of the disease in Korea in 2015 [9].

At the time of the initial COVID-19 outbreak, the Korean infection control authorities decided to designate certain medical facilities for the rapid treatment of confirmed COVID-19 patients with a mild, low-severity disease course, in accordance with the country's Infectious Disease Control Act [10,11]. As a result, numerous hospitals have been fully designated as centers for COVID-19. A designated facility, according to the Infectious Disease Control Act, uses existing beds to treat moderately ill confirmed patients. If patients' condition deteriorates, they are immediately transferred to a nearby university hospital equipped with intensive care unit (ICU) facilities. Therefore, among the inpatients of the designated facilities analyzed herein, there were few severely ill patients, and an ICU for COVID-19 patients was not operated. However, a great deal of stress was imposed on the hospital employees related to the inconvenience of caring for inpatients in isolated wards, wearing protective equipment, as well as fear and social stigma of being a potential source of infection.

During the COVID-19 pandemic, all employees, including those working at non-medical departments, were engaged in screening and inpatient treatment, which were duties of the designated hospitals. Employees who were working daytime shifts also began working night-time shifts and were required to carry out screening and ward tasks. Employees were also assigned to work modified shifts (2 shifts per week, working every other day, etc.). Furthermore, some employees working in administrative departments (such as the general affairs department or finance department) took turns performing quarantine-related tasks, such as medical waste disposal.

There have been conflicting reports about the mental health of medical and non-medical workers at hospitals. A study reported that medical workers showed a higher likelihood of anxiety symptoms than non-medical workers [12]. Other studies showed that psychological risk was higher among nonmedical workers [13] and that $50.3 \%$ of frontline non-medical workers complained of depressive symptoms [14].

According to the literature on facilities designated as COVID-19 care centers, centralized coordination of frontline hospital operations, staff management, and patient treatment and placement made it possible to successfully assemble and utilize medical resources and personnel during the COVID-19 outbreak [15]. Since the COVID-19 pandemic is expected to continue for a long time, the operation of facilities dedicated to a specific infectious disease such as COVID-19 is thought to be a useful measure. There is also a clear need for research on the mental health of both medical and non-medical personnel at designated treatment facilities.

Korea is one of the few countries around the world that experienced the MERS outbreak prior to the outbreak of COVID-19. Therefore, a significant number of healthcare workers and hospital officials working to prevent the current spread of COVID-19 had previously experienced the MERS outbreak in Korea. Several studies have examined the psychological impact of previous outbreaks on healthcare workers $[9,16]$. However, only a few considered the impact of previous experiences with an outbreak (e.g., Middle East respiratory syndrome coronavirus [MERS-CoV] or SARS-CoV) on the mental health of healthcare workers in the current COVID-19 situation. Healthcare workers at tertiary care teaching hospitals tended to experience significant levels of anxiety and stress during the $\mathrm{CO}$ VID-19 pandemic, which impaired their attention, cognitive functioning, and clinical decision-making [17]. Anticipation regarding subsequent waves of infection reflects the possibility of a prolonged pandemic. Consequently, the stress of hospital workers engaged in quarantine is also expected to last for a long time. Therefore, gaining an understanding of how the current situation affects the mental health of hospital workers who experienced the previous epidemic is urgent to enable timely interventions.

Additionally, unlike the previous outbreak, the current pre- 
ventive system for COVID-19 expects participation not only from frontline medical personnel, such as physicians or nurses, but also from non-medical personnel who are not in direct contact with infected patients. Since every worker in the hospital is involved, at least to some extent, in the preventive systems of quarantine, understanding the effect of occupational type beyond healthcare professionals on mental health during this pandemic is essential.

This study investigated the association between the prevalence of PTSD and various perceptions of COVID-19 among medical and non-medical personnel at medical centers. Additionally, this study examined general perceptions about COVID-19 according to occupational type and previous experience of the MERS outbreak in order to understand the relationship of those factors to the prevalence of PTSD.

\section{METHODS}

\section{Data and Participants}

Data were collected using a questionnaire targeting all employees, including hospital administration and medical personnel, working at medical facilities designated for COVID-19 located in different cities of South Chungcheong Province (nameIy, Cheonan, Seosan, Gongju, and Hongseong) in Korea. The questionnaire was designed to investigate perceived fear, perceived discomfort due to shortages of supplies, the perceived severity of COVID-19, and PTSD ascertained by Posttraumatic Stress Disorder Checklist for the Diagnostic and Statistical Manual of Mental Disorders, 5th edition (PCL-5) [18]. From an original pool of 631 targeted workers invited to participate in the survey through text messages, 397 workers were interviewed (response rate, 62.9\%). However, data obtained from 1 worker were excluded from the analysis due to missing information regarding relevant questions (e.g., sex). Consequently, data obtained from 396 hospital workers (78 male and 318 female) were available for analysis (Figure 1).

\section{Study Tool}

The survey questionnaire covered the socio-demographic characteristics; perceived fear related to income, the burden of family care duties, and social stigma due to COVID-19 infection; perceived discomfort due to shortages of supplies (foods, necessities, masks, disinfectant, etc.) caused by the pandemic; and the perceived disease severity of COVID-19. Each question asked participants to rate their response on a Likert scale, ranging

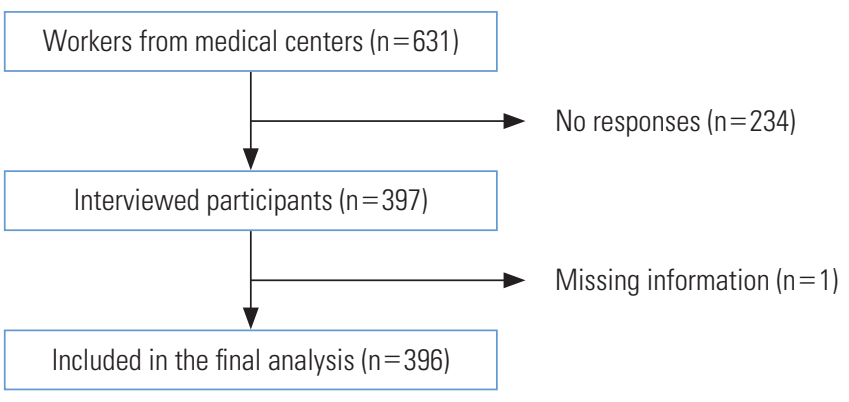

Figure 1. Flow chart of participant enrollment.

from 0 (not at all) to 5 (very much so). The survey also contained validated tools to measure various symptoms related to mental health, such as depression, anxiety, and PTSD, ascertained by the PCL-5 with a cut-off value of 33 [18].

The questionnaire used in this study was adopted in part from the content of the risk perception items used in earlier studies that investigated various mental health indicators in relation to the earlier SARS outbreak [19-21]. Assessments to establish the validity and reliability of the questionnaire were conducted. Initially, 3 experts in the field of epidemiology and occupational environmental medicine were asked to assess the degree to which the items in the questionnaires were relevant and could correctly measure perceptions of fear, discomfort, and disease severity among hospital workers regarding COVID-19. Duplicated items were compressed, and items related to the current pandemic were added. The items included loss of income, burden of family care duties, and fear of social stigma due to the possibility of infection. Finally, an item measuring the perceived overall fear and disease severity of COVID-19 was also included. In consideration of non-medical participants, the survey was prepared using non-technical terms as much as possible.

\section{Validation}

Primary component analysis was performed to verify the validity of the items. Supplementary Material 1 is a summary of the eigenvectors obtained by principal component analysis (PCA) of 9 items measured on a 6-point Likert scale to investigate perceptions. According to the results shown on a scree plot in Supplementary Material 2, the items were classified using the eigenvector up to principal component 3 (PC3). The PCA results demonstrated that the items could be classified as questions about specific fears of possible income loss, burden of family care duties, and social stigma due to possible infection ("issue-specific fear"); recognition of shortages of food, 
daily necessities, hygiene products, and quarantine supplies ("discomfort due to shortages of supplies"); and overall fear and disease severity of COVID-19 ("general fear").

Based on these results, confirmatory factor analysis (CFA) was conducted. In order to measure the construct validity, indicators such as standard loading, average variance extracted (AVE), and construct reliability (CR) were calculated. Supplementary Material 3 is a table that summarizes the results of CFA. The standard estimates were 0.5 or higher in most cases; and the AVE and CR were 0.54 and 0.82 , respectively, for perceived fear; 0.18 and 0.40 , respectively, for perceived discomfort due to shortages of supplies; and 0.72 and 0.81 , respectively, for perceived fear and disease severity toward COVID-19. The analysis was conducted using the "lavaan" package of $\mathrm{R}$ version 3.6.3 (https://cran.r-project.org/).

The reliability of the study tool was evaluated by surveying 30 participants of the sample. Data were used to assess the internal reliability using the Cronbach alpha coefficient. The results showed adequate internal reliability (with Cronbach alpha $=0.80$ for fear due to income, family care, and stigma; 0.80 for perceived discomfort due to shortages of supplies, such as food, necessities, hygiene products, and quarantine supplies caused by COVID-19; and 0.70 for perceived fear and severity of (OVID-19 as a disease).

\section{Covariates}

The covariates consisted of age, sex, marital status, and chronic disease prevalence. These were adjusted and analyzed to clarify the associations of the independent and dependent variables. Participants with chronic diseases were classified as those with 1 or more of the following conditions: pre-diagnosed hypertension, diabetes, dyslipidemia, cardiovascular disease, thyroid-related disease, and malignant tumor. To analyze the association of PTSD prevalence according to occupational type, the participants were stratified into medical personnel, including physicians and nurses, and non-medical personnel, including other occupations. Additionally, participants were stratified into 2 groups based on their experience of conducting preventive work during previous coronavirus outbreaks, such as MERS.

\section{Statistical Analysis}

Participants' characteristics, some of which were converted into categorical variables, were compared according to PTSD status using the chi-square test (Table 1). Multivariable logistic
Table 1. General characteristics of participants by PTSD classification ${ }^{1}$

\begin{tabular}{|c|c|c|c|c|}
\hline \multirow{2}{*}{ Characteristics } & \multirow{2}{*}{$\begin{array}{c}\text { Total } \\
(n=396)\end{array}$} & \multicolumn{2}{|c|}{ PTSD } & \multirow{2}{*}{$p$-value } \\
\hline & & Yes $(n=53)$ & No $(n=343)$ & \\
\hline Age (y) & & & & 0.726 \\
\hline $20-29$ & $133(33.6)$ & $16(12.0)$ & $117(88.0)$ & \\
\hline $30-39$ & $119(30.0)$ & $14(11.8)$ & $105(88.2)$ & \\
\hline $40-49$ & $88(22.2)$ & $14(15.9)$ & $74(84.1)$ & \\
\hline$\geq 50$ & $56(14.1)$ & $9(16.1)$ & 47 (83.9) & \\
\hline Sex & & & & 0.563 \\
\hline Male & $78(19.7)$ & $12(15.4)$ & $66(84.6)$ & \\
\hline Female & $318(80.3)$ & 41 (12.9) & $277(87.1)$ & \\
\hline Marital status & & & & 0.776 \\
\hline Never & $187(47.2)$ & $23(12.3)$ & $164(87.7)$ & \\
\hline Married, living together & $198(50.0)$ & $28(7.1)$ & $170(85.9)$ & \\
\hline $\begin{array}{l}\text { Married, divorced or } \\
\text { widowed }\end{array}$ & $11(2.8)$ & 2 (18.2) & $9(81.8)$ & \\
\hline Occupation & & & & 0.987 \\
\hline $\begin{array}{c}\text { Medical personnel } \\
\text { (doctor or nurse) }\end{array}$ & $306(77.3)$ & 41 (13.4) & 265 (86.6) & \\
\hline Others & $90(22.7)$ & $12(13.3)$ & 78 (86.7) & \\
\hline Prior MERS experience & & & & 0.025 \\
\hline Yes & $161(40.7)$ & $29(18.0)$ & $132(82.0)$ & \\
\hline No & $235(59.3)$ & $24(10.2)$ & $211(89.8)$ & \\
\hline Chronic disease $^{2}$ & & & & 0.034 \\
\hline 0 & $337(85.1)$ & $40(11.9)$ & $297(88.1)$ & \\
\hline$\geq 1$ & $59(14.9)$ & $13(22.0)$ & 46 (78.0) & \\
\hline
\end{tabular}

Values are presented as number (\%).

PTSD, posttraumatic stress disorder; MERS, Middle East respiratory syndrome.

1PTSD was defined by using the PTSD Checklist for the Diagnostic and Statistical Manual of Mental Disorders, 5th edition with a cut-off of 33, validated by Bovin et al. [18].

${ }^{2}$ Chronic diseases included hypertension, dyslipidemia, diabetes, cardiovascular disease, thyroid-related disease, and malignancy.

regression, adjusted for the aforementioned covariates, was used to evaluate the associations of perceived specific fear regarding income, family care burden, social stigma; discomfort due to shortages of supplies; and overall fear and severity of COVID-19 with the prevalence of PTSD (Table 2). The technique was also used to model a stratified analysis according to occupational type and prior MERS-CoV experience, as shown in Figures 2 and 3. For statistical analysis, SAS version 9.4 (SAS Institute Inc., Cary, NC, USA) was used, and the statistical significance level was set at 0.05 .

\section{Ethics Statement}

The Institutional Review Board of Yonsei University Health System, Seoul, Korea approved the study protocol (COVID-19 
Table 2. Associations between general perceptions of COVID-19 and PTSD $(n=396)^{1}$

\begin{tabular}{|c|c|c|}
\hline \multirow{2}{*}{ Perception [range] ${ }^{2,3}$} & \multicolumn{2}{|c|}{ PTSD $(n=53)$} \\
\hline & OR $(95 \% \mathrm{Cl})^{4,5}$ & $p$-value \\
\hline \multicolumn{3}{|l|}{ Total $(n=396)$} \\
\hline Issue-specific fear [0-15] & $1.18(1.08,1.28)$ & $<0.001$ \\
\hline Discomfort due to shortages of supplies [0-20] & $1.12(1.05,1.20)$ & 0.001 \\
\hline General fear [0-10] & $1.56(1.25,1.94)$ & $<0.001$ \\
\hline \multicolumn{3}{|l|}{ Occupational type $(n=396)$} \\
\hline \multicolumn{3}{|l|}{ Medical personnel $(n=306)$} \\
\hline Issue-specific fear [0-15] & $1.16(1.05,1.28)$ & 0.003 \\
\hline Discomfort due to shortages of supplies [0-20] & $1.08(1.00,1.16)$ & 0.059 \\
\hline General fear [0-10] & $1.37(1.08,1.74)$ & 0.009 \\
\hline \multicolumn{3}{|l|}{ Non-medical personnel $(n=90)$} \\
\hline Issue-specific fear [0-15] & $1.29(1.05,1.59)$ & 0.017 \\
\hline Discomfort due to shortages of supplies [0-20] & $1.29(1.07,1.56)$ & 0.008 \\
\hline General fear [0-10] & $6.67(1.92,23.20)$ & 0.003 \\
\hline \multicolumn{3}{|l|}{ Previous MERS-CoV experience ( $n=396)$} \\
\hline \multicolumn{3}{|l|}{ No experience of MERS ( $n=235)$} \\
\hline Issue-specific fear [0-15] & $1.15(1.03,1.29)$ & 0.017 \\
\hline Discomfort due to shortages of supplies [0-20] & $1.04(0.94,1.14)$ & 0.475 \\
\hline General fear [0-10] & $1.45(1.06,2.00)$ & 0.021 \\
\hline \multicolumn{3}{|l|}{ Experience of MERS ( $n=161)$} \\
\hline Issue-specific fear [0-15] & $1.21(1.06,1.38)$ & 0.021 \\
\hline Discomfort due to shortages of supplies [0-20] & $1.24(1.10,1.40)$ & 0.005 \\
\hline General fear [0-10] & $1.70(1.22,2.37)$ & 0.002 \\
\hline
\end{tabular}

COVID-19, coronavirus disease 2019; PTSD, posttraumatic stress disorder; $\mathrm{OR}$, odds ratio; $\mathrm{Cl}$, confidence interval; MERS-CoV, Middle East respiratory syndrome coronavirus; MERS, Middle East respiratory syndrome.

'PTSD was defined by using the PTSD Checklist for Diagnostic and Statistical Manual of Mental Disorders, 5th edition with a cut-off of 33, validated by Bovin et al. [18].

'Each composite score consisted of items asking about participants' perceptions about COVID-19, with responses on a Likert scale ranging from 0 (not at all) to 5 (very much so).

${ }^{3}$ The issue-specific fear domain consisted of items asking about specific fears on possible income loss, burden of family care duties, and social stigma due to possible infection; The discomfort due to shortages of supplies domain consisted of items asking about shortages of food, daily necessities, hygiene products, and quarantine supplies; The general fear domain consisted of items asking about overall fear and perceived disease severity of COVID-19.

${ }^{4}$ Models adjusted for age, sex, marital status, and chronic disease.

${ }^{5}$ Per 1 unit increase.

mental health survey: Y-2020-0034). Written consent was obtained from all participants before the evaluation and survey. All procedures contributing to this work complied with the ethical standards of the relevant national and institutional committees on human experimentation and with the Helsinki Declaration (2013).

\section{RESULTS}

Supplementary Material 4 summarizes the distribution of participants according to occupational type. The occupations of the COVID-19-designated hospital workers who were selected as participants in this study were as follows: nurses $(\mathrm{n}=$ $291,73.5 \%)$, nursing assistants $(n=25,6.3 \%)$, physical therapists $(n=6,1.5 \%)$, radiology technicians $(n=6,1.5 \%)$, doctors $(n=15,3.8 \%)$, clinical pathology technicians $(n=5,1.3 \%)$, administrative department staff $(n=13,3.3 \%)$, finance department staff $(n=14,3.5 \%)$, and other employees $(n=21,5.3 \%)$. Doctors and nurses, who had direct contact with patients confirmed with COVID-19, were categorized as medical personnel $(n=306)$, and workers in the remaining occupations were categorized as non-medical personnel $(n=90)$.

Supplementary Material 5 summarizes the degree of direct or indirect exposure of each medical center employee to confirmed COVID-19 patients. The proportion of direct contact with confirmed patients was higher for the medical personnel. However, even among the non-medical personnel, a significant number $(n=31,34.4 \%)$ of employees showed the highest level of direct contact.

Table 1 shows the general characteristics of 396 participants according to the prevalence of PTSD. There were no significant differences in demographic characteristics, such as age, sex, marital status, and occupation, except for previous MERS experience $(p<0.05)$ and chronic disease $(p<0.05)$.

Table 2 summarizes the associations between the prevalence of PTSD measured by PCL-5 and perceived fear, discomfort due to shortage of supplies, and overall fear and severity of the disease, as well as the results of the stratified analyses. The prevalence of PTSD tended to increase with a 1-unit increment for all domains in the questionnaire regarding perceptions. As the score for issue-specific fear increased by 1 unit, the odds of being classified as having PTSD also increased (odds ratio [OR], 1.18; 95\% confidence interval [Cl], 1.08 to 1.28$)$. The association between perceived discomfort due to shortages of supplies and being classified as having PTSD was also statistically significant (OR, 1.12; $95 \% \mathrm{Cl}, 1.05$ to 1.20). Furthermore, an increase in the score for general fear was significantly associated with higher odds of being classified as having PTSD (OR, 1.56; $95 \% \mathrm{Cl}, 1.25$ to 1.94$)$.

Figure 2 is a plot showing the associations of issue-specific fear, discomfort due to shortages of supplies, and general fear with prevalence of PTSD by stratifying participants into medi- 


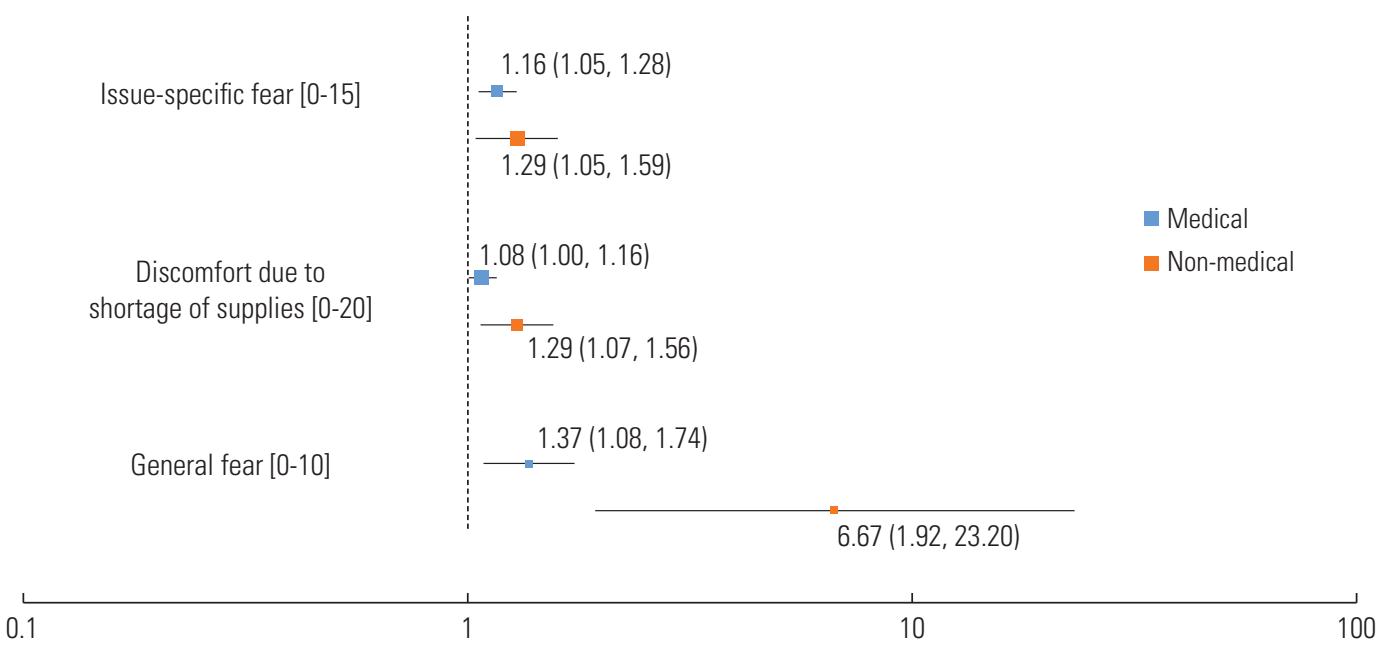

Figure 2. Association between perception and posttraumatic stress disorder prevalence stratified by occupation type. The size of the indicator reflects the standard error (SE) $\left(\sim 1 / \mathrm{SE}^{2}\right)$. Values are presented as odds ratio (95\% confidence interval).

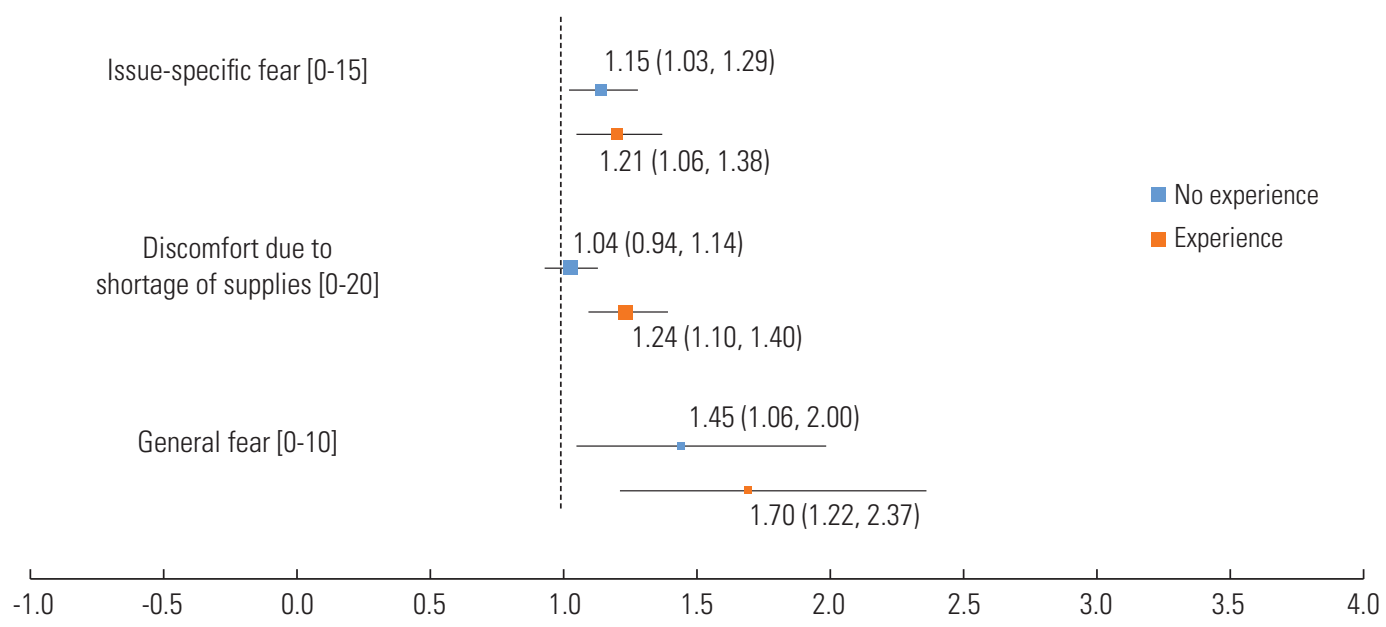

Figure 3. Association between perception and posttraumatic stress disorder prevalence stratified by previous Middle East respiratory syndrome experience. The size of the indicator reflects the standard error (SE) $\left(\sim 1 / \mathrm{SE}^{2}\right)$. Values are presented as odds ratio (95\% confidence interval).

cal personnel and non-medical personnel. The $x$-axis represents the OR, which is plotted on a logarithmic scale. In general, nonmedical personnel had higher odds of having PTSD for all domains related to perceptions than medical personnel.

Figure 3 presents the results of associations between perceptions and PTSD prevalence. Participants were stratified by their previous experience of the MERS outbreak. For all domains, the group with prior MERS experience tended to show higher odds of having PTSD than the group without previous experience. Except for discomfort due to shortages of supplies (OR, 1.04; $95 \% \mathrm{Cl}, 0.94$ to 1.14), all other domains showed statistically significant associations with the odds of having PTSD.

\section{DISCUSSION}

Our study investigated the associations of perceived fear, discomfort, and severity of COVID-19 with PTSD prevalence among hospital workers at 4 distinct medical centers that were designated for COVID-19 patients. The following are the key findings of this study: (1) All domains associated with perceptions-fear specific to income, family care burden, and social stigma; discomfort due to shortage of supplies; and overall fear and severity of COVID-19-were associated with hospital workers' odds of being classified as having PTSD (Table 2). (2) The magnitude of these associations was greater among non- 
medical personnel than among medical personnel. (3) The magnitude of associations between the prevalence of PTSD and the general perceptions about COVID-19 tended to be higher in those who had experience with the previous MERS outbreak than among those without prior experience.

Our results showed the impact of previous MERS-related experience on healthcare workers during the COVID-19 outbreak. Previous studies have also investigated the impact of MERS$\mathrm{CoV}$ on the mental health of current medical personnel, and the results were consistent with our results to some extent. A study conducted among healthcare workers at tertiary care teaching hospitals in Saudi Arabia [17] reported that infectious diseases, such as COVID-19 or MERS-CoV, trigger a significant level of stress in healthcare workers, with the concern being that the nature of the workplace involves more frequent direct contact with confirmed patients and, therefore, a risk of transmitting the virus to their families. This accounts for the higher rate of being classified as having PTSD among the participants in our study, who were workers at hospitals designated to treat COVID-19, than the general prevalence rate.

In a cross-sectional study on healthcare workers in Singapore including non-medical personnel, such as pharmacists, technicians, and administrators [13], the authors reported that nonmedical workers had higher anxiety levels than medical personnel. In a study involving 214 people from the general public and 526 nurses aiding in COVID-19 control (234 frontline nurses and 292 non-frontline nurses) [22], non-frontline nurses had higher vicarious trauma scores than did frontline nurses. The results of these existing studies are consistent with our findings in that the magnitude of the relationship was stronger among non-medical workers. The reasons for this may include a relatively higher fear toward the uncertain contagiousness of the disease, less medical information, and less-intensive training [22] in personal protective equipment and infection control measures [13]. Moreover, our results reporting a higher OR among non-medical workers than among medical workers for being classified as having PTSD due to concerns regarding daily necessities, family care burden, and quarantine supplies may also be explained by the fact that, in the context of the economic downturn caused by the COVID-19 pandemic, employment security is less stable for non-medical personnel [23,24].

PTSD is a mental disorder that is characterized by excessive fear responses to potential threats and attempts to avoid trauma-related cues. Animal research on fear conditioning has provided support to anatomical models of PTSD, suggesting that exaggerated amygdala responses to fear reflect a lack of descending inhibition in the medial prefrontal cortex $[25,26]$. Psychological distress levels may be exacerbated not only by the fear of being infected, but also by the fear of becoming a carrier of the virus, causing transmission among fellow healthcare workers and one's own family [27]. Distress can also be reinforced by fears of reduced income due to job loss and social stigma as a result of contracting the infection [27]. Shortages of daily necessities or preventive goods, such as face masks, can also trigger psychopathological fear and anxiety responses [28].

Previous traumatizing events, such as a disaster, accidents, or an epidemic, have been found to influence the fear response of potential victims [29-32]. Previous experiments with rodents consistently observed that rats exposed to an intense electrical shock not only showed a strong fear response when they were re-exposed to the shock, but also displayed fear-like responses when exposed to a novel environment unrelated to the previous exposure [33-35].

A study of employees at 3 hospitals affected by a severe snowstorm reported that the disaster-related experience changed the relationships among perceptions of sociotechnical safety factors [35]. Individuals tended to perceive risks as having greater effects if they had previously experienced a disaster than if they had not. Therefore, healthcare workers who experienced an epidemic in the past may perceive more stress.

This study had several limitations. First, since this study was cross-sectional, the ability to derive causal inferences from the associations between the perceptions of COVID-19 and the prevalence of PTSD is limited. Similarly, this limitation also implies the possibility of a reverse-causation relationship between general perceptions of COVID-19 and the prevalence of PTSD. Second, the overall response rate of this study was $62.9 \%$ (397 of 631), which might have led to selection bias. Potential participants were sent a request to fill out a questionnaire, meaning that workers with a heavy workload might have been excluded. The response rates were $60.0 \%$ for doctors (15 of 25 ), $66.7 \%$ for nurses ( 298 of 447 ), and $52.8 \%$ for other occupations (84 of 159). We intended to include all hospital workers from 3 nearby medical centers. However, due to differences in the level of cooperation within each department, workers from the nursing department were disproportionately enrolled in the registration process. Third, our sample was extracted using convenience selection. It is difficult for a sample obtained using this method to have representativeness. Since this study 
aimed at deriving an initial result regarding the mental health of healthcare workers in the region, a sampling method encompassing representatives of hospital workers nationwide must be used in follow-up studies. Fourth, the PCL-5 used in this study is a screening tool for diagnosing PTSD. A definitive tool, such as the Clinician-Administered Posttraumatic Stress Disorder scale for the Diagnostic and Statistical Manual of Mental Disorders, 5th edition, should have been used for a more reliable diagnosis. However, the PCL-5 shows good internal consistency ( $\alpha=0.96)$, test-retest reliability, and convergent and discriminant validity [18]. Finally, the possibility of residual confounding remains, as not all variables considered as confounders were measured and included in the model.

Regardless of its limitations, this study was conducted among medical and non-medical workers at facilities designated for confirmed COVID-19 patients. Many researchers have investigated the mental health of hospital workers, including nonmedical personnel, using factors such as their sleep quality, somatic symptoms, depression, and anxiety $[12,36,37]$. This study provided additional data regarding the mental health issues of both medical and non-medical workers who are dedicated to treat confirmed cases of COVID-19. During the COVID-19 pandemic, since authorities expect the participation of both healthcare workers and members of the community in quarantine, the effect of stress caused by COVID-19 on nonmedical personnel should not be overlooked.

Moreover, Korea is one of the few countries around the world that experienced the MERS-CoV outbreak prior to the outbreak of COVID-19. As a result, a significant number of healthcare workers in Korea experienced quarantine during the MERS outbreak. We included a rare sample that enabled us to study the psychological impact of previous MERS experience on the mental health of medical and non-medical personnel during the current COVID-19 pandemic. Our study, therefore, is a valuable source for understanding the effects of previous epidemic experiences on the mental health of healthcare workers dedicating themselves to combatting the current pandemic.

Non-medical healthcare personnel were shown to be at higher risk of PTSD than medical personnel during the COVID-19 outbreak. Previous MERS experience exacerbated the risk of PTSD. Early psychological monitoring and interventions for hospital workers may be beneficial, especially for non-medical personnel. Educational interventions and training should be given to non-medical healthcare workers to ensure their understanding of infectious disease control measures.

\section{SUPPLEMENTAL MATERIALS}

Supplemental materials are available at https://doi.org/10. 3961/jpmph.20.540.

\section{CONFLICT OF INTEREST}

The authors have no conflicts of interest associated with the material presented in this paper.

\section{FUNDING}

This study was supported by National Research Foundation of Korea, funded by the Ministry of Science and ICT (2020R1C1C1003502).

\section{ACKNOWLEDGEMENTS}

The authors would like to thank the Medical Illustration and Design department, which is part of the Medical Research Support Services of Yonsei University College of Medicine, for all of the artistic support related to this study.

\section{AUTHOR CONTRIBUTIONS}

Conceptualization: YL, SJJ. Data curation: SP. Formal analysis: YL, KK, SJJ. Funding acquisition: SJJ. Methodology: YL, SJJ. Project administration: SJJ. Visualization: YL. Writing - original draft:YL. Writing - review \& editing: YL, SP, KK, SJJ.

\section{ORCID}

Youngrong Lee https://orcid.org/0000-0003-1367-4381

Kwanghyun Kim https://orcid.org/0000-0001-9552-5085

Sungjin Park https://orcid.org/0000-0002-9240-9684

Sun Jae Jung https://orcid.org/0000-0002-5194-7339

\section{REFERENCES}

1. Chen N, Zhou M, Dong X, Qu J, Gong F, Han Y, et al. Epidemiological and clinical characteristics of 99 cases of 2019 novel coronavirus pneumonia in Wuhan, China: a descriptive study. Lancet 2020;395(10223):507-513.

2. Johns Hopkins Department of Civil and Systems Engineering. COVID-19 map FAQs [cited 2020 May 1]. Available from: https:// 
systems.jhu.edu/research/public-health/2019-ncov-map-faqs/.

3. Leung K, Wu JT, Liu D, Leung GM. First-wave COVID-19 transmissibility and severity in China outside Hubei after control measures, and second-wave scenario planning: a modelling impact assessment. Lancet 2020;395(10233):1382-1393.

4. Pappa S, Ntella V, Giannakas T, Giannakoulis VG, Papoutsi E, Katsaounou P. Prevalence of depression, anxiety, and insomnia among healthcare workers during the COVID-19 pandemic: a systematic review and meta-analysis. Brain Behav Immun 2020;88:901-907.

5. Wong TW, Yau JK, Chan CL, Kwong RS, Ho SM, Lau CC, et al. The psychological impact of severe acute respiratory syndrome outbreak on healthcare workers in emergency departments and how they cope. Eur J Emerg Med 2005;12(1):13-18.

6. Greenberg N, Docherty M, Gnanapragasam S, Wessely S. Managing mental health challenges faced by healthcare workers during covid-19 pandemic. BMJ 2020;368:m1211.

7. Litz BT, Stein N, Delaney E, Lebowitz L, Nash WP, Silva C, et al. Moral injury and moral repair in war veterans: a preliminary model and intervention strategy. Clin Psychol Rev 2009;29(8): 695-706.

8. Torales J, O'Higgins M, Castaldelli-Maia JM, Ventriglio A. The outbreak of COVID-19 coronavirus and its impact on global mental health. Int J Soc Psychiatry 2020;66(4):317-320.

9. Lee SM, Kang WS, Cho AR, Kim T, Park JK. Psychological impact of the 2015 MERS outbreak on hospital workers and quarantined hemodialysis patients. Compr Psychiatry 2018;87:123127.

10. Ministry of Health and Welfare. (3.7.) Regular briefing of central disaster and safety countermeasure headquarters on $\mathrm{CO}$ VID-19; 2020 Mar 7 [cited 2020 Dec 22]. Available from: http:// www.mohw.go.kr/eng/nw/nw0101vw.jsp?PAR_MENU_ID= $1007 \& M E N U$ ID $=100701 \&$ page $=3 \& C O N T$ SEQ $=353495 \&$ SE ARCHKEY $=$ TITLE\&SEARCHVALUE $=$ covid.

11. Ministry of Health and Welfare. 91 Medical institutions designated as "National Relief Hospital" [cited 2020 Dec 22 ]. Available from: http://www.mohw.go.kr/react/al/sal0301vw.jsp? PAR_MENU_ID $=04 \& M E N U \_I D=0403 \&$ page $=2 \& C O N T \_S E Q$ $=353148 \&$ SEARCHKEY $=$ TITLE\&SEARCHVALUE $=\% E C \% A 7 \% 8$ 0\%EC\%A0\%95 (Korean).

12. Maciaszek J, Ciulkowicz M, Misiak B, Szczesniak D, Luc D, Wieczorek T, et al. Mental health of medical and non-medical professionals during the peak of the COVID-19 pandemic: a crosssectional nationwide study. J Clin Med 2020;9(8):2527.

13. Tan BY, Chew NW, Lee GK, Jing M, Goh Y, Yeo LL, et al. Psycho- logical impact of the COVID-19 pandemic on health care workers in Singapore. Ann Intern Med 2020;173(4):317-320.

14. Fang X, Zhang J, Teng C, Zhao K, Su KP, Wang Z, et al. Depressive symptoms in the front-line non-medical workers during the COVID-19 outbreak in Wuhan. J Affect Disord 2020;276: 441-445.

15. Kim M, Lee JY, Park JS, Kim HA, Hyun M, Suh YS, et al. Lessons from a COVID-19 hospital, Republic of Korea. Bull World Health Organ 2020;98(12):842-848.

16. Park JS, Lee EH, Park NR, Choi YH. Mental health of nurses working at a government-designated hospital during a MERS-CoV outbreak: a cross-sectional study. Arch Psychiatr Nurs 2018; 32(1):2-6.

17. Temsah MH, Al-Sohime F, Alamro N, Al-Eyadhy A, Al-Hasan K, Jamal A, et al. The psychological impact of COVID-19 pandemic on health care workers in a MERS-CoV endemic country. J Infect Public Health 2020;13(6):877-882.

18. Bovin MJ, Marx BP, Weathers FW, Gallagher MW, Rodriguez $P$, Schnurr PP, et al. Psychometric properties of the PTSD Checklist for Diagnostic and Statistical Manual of Mental DisordersFifth Edition (PCL-5) in veterans. Psychol Assess 2016;28(11): 1379-1391.

19. Wu P, Fang Y, Guan Z, Fan B, Kong J, Yao Z, et al. The psychological impact of the SARS epidemic on hospital employees in China: exposure, risk perception, and altruistic acceptance of risk. Can J Psychiatry 2009;54(5):302-311.

20. Long NN, Khoi BH. An empirical study about the intention to hoard food during COVID-19 pandemic. EURASIA J Math Sci Tech Ed 2020;16(7):em1857.

21. Wen Z, Huimin G, Kavanaugh RR. The impacts of SARS on the consumer behaviour of Chinese domestic tourists. Curr Issues Tour 2005;8(1):22-38.

22. Li Z, Ge J, Yang M, Feng J, Qiao M, Jiang R, et al. Vicarious traumatization in the general public, members, and non-members of medical teams aiding in COVID-19 control. Brain Behav Immun 2020;88:916-919.

23. Rönnblad T, Grönholm E, Jonsson J, Koranyi I, Orellana C, Kreshpaj B, et al. Precarious employment and mental health: a systematic review and meta-analysis of longitudinal studies. Scand J Work Environ Health 2019;45(5):429-443.

24. Wilson JM, Lee J, Fitzgerald HN, Oosterhoff B, Sevi B, Shook NJ. Job Insecurity and financial concern during the COVID-19 pandemic are associated with worse mental health. J Occup Environ Med 2020;62(9):686-691.

25. Milad MR, Quirk GJ. Neurons in medial prefrontal cortex signal 
memory for fear extinction. Nature 2002;420(6911):70-74.

26. Morgan MA, Romanski LM, LeDoux JE. Extinction of emotional learning: contribution of medial prefrontal cortex. Neurosci Lett 1993;163(1):109-113.

27. Chew NW, Lee GK, Tan BY, Jing M, Goh Y, Ngiam NJ, et al. A multinational, multicentre study on the psychological outcomes and associated physical symptoms amongst healthcare workers during COVID-19 outbreak. Brain Behav Immun 2020; 88:559-565.

28. Wang C, Pan R, Wan X, Tan Y, Xu L, Ho CS, et al. Immediate psychological responses and associated factors during the initial stage of the 2019 coronavirus disease (COVID-19) epidemic among the general population in China. Int J Environ Res Public Health 2020;17(5):1729.

29. Chen X, Li Y, Li S, Kirouac GJ. Early fear as a predictor of avoidance in a rat model of post-traumatic stress disorder. Behav Brain Res 2012;226(1):112-117.

30. Stam R. PTSD and stress sensitisation: a tale of brain and body: part 1: human studies. Neurosci Biobehav Rev 2007;31(4): 530-557.

31. Davidson JR, Stein DJ, Shalev AY, Yehuda R. Posttraumatic stress disorder: acquisition, recognition, course, and treatment. J Neuropsychiatry Clin Neurosci 2004;16(2):135-147.

32. Bonne O, Grillon C, Vythilingam M, Neumeister A, Charney DS.
Adaptive and maladaptive psychobiological responses to severe psychological stress: implications for the discovery of novel pharmacotherapy. Neurosci Biobehav Rev 2004;28(1): 65-94.

33. Daviu N, Fuentes S, Nadal R, Armario A. A single footshock causes long-lasting hypoactivity in unknown environments that is dependent on the development of contextual fear conditioning. Neurobiol Learn Mem 2010;94(2):183-190.

34. Siegmund A, Wotjak CT. A mouse model of posttraumatic stress disorder that distinguishes between conditioned and sensitised fear. J Psychiatr Res 2007;41(10):848-860.

35. Park I, Sharman R, Rao HR. Disaster experience and hospital information systems: an examination of perceived information assurance, risk, resilience, and his usefulness. MIS Q 2015;39(2): 317-344.

36. Wang W, Song W, Xia Z, He Y, Tang L, Hou J, et al. Sleep disturbance and psychological profiles of medical staff and non-medical staff during the early outbreak of COVID-19 in Hubei Province, China. Front Psychiatry 2020;11:733.

37. Chen B, Li QX, Zhang H, Zhu JY, Yang X, Wu YH, et al. The psychological impact of COVID-19 outbreak on medical staff and the general public. Curr Psychol 2020. doi: https://doi.org/10. 1007/s12144-020-01109-0. 